Elucidation of signaling pathways that regulate ethylene-induced leaf and flower abscission of agriculturally important plants

Tucker, M.L. USDA, Agricultural Research Service MD

Meir, S. Agricultural Research Organization

Lers, A. Agricultural Research Organization

Philosoph-Hadas, S. Agricultural Research Organization

Jiang, C. USDA, Agricultural Research Service CA

Project award year: 2012

Three year research project 


\begin{abstract}
The Problem: Abscission is a highly regulated process, occurring as a natural terminal stage of development, in which various organs are separated from the parent plant. In most plant species, the process is initiated by a decrease in active auxin in the abscission zone (AZ) and an increase in ethylene, and may be accelerated by postharvest or environmental stresses. Another potential key regulator in abscission is IDA (Inflorescence Deficient in Abscission), which was identified as an essential peptide signal for floral organ abscission in Arabidopsis. However, information is still lacking regarding the molecular mechanisms integrating all these regulators. In our previous BARD funded research we made substantial progress towards understanding these molecular events in tomato, and the study is still in progress. We established a powerful platform for analysis of genes for regulatory proteins expressed in AZ. We identified changes in gene expression for several transcription factors (TFs) directly linked to ethylene and auxin signaling and several additional regulatory proteins not so obviously linked to these hormones. Moreover, we demonstrated using a virus-induced gene silencing (VIGS) assay that several play a functional role in the onset of abscission. Based on these results we have selected 14 genes for further analysis in stably transformed tomato plants. All 14 genes were suppressed by RNA interference (RNAi) using a constitutive promoter, and 5 of them were also suppressed using an abscission-specific promoter. Transformations are currently at different stages of progress including some lines that already display an abscission phenotype. Objectives: We propose here to (1) complete the functional analysis of the stably transformed tomato plants with T2 lines and perform transcriptome analysis using custom abscission-specific microarrays; (2) conduct an indepth analysis of the role of IDA signaling in tomato leaf and flower abscission; (3) perform transcriptome and proteome analyses to extend the earlier gene expression studies to identify transcripts and proteins that are highly specific to the separation layer (i.e., target cells for cell separation) prior to the onset of abscission; (4) extend and compliment the work in tomato using a winnowed set of genes in soybean. Methodology: Next Generation Sequencing (NGS) of mRNA will be used to further increase the list of abscission-associated genes, and for preparation of a custom tomato abscission microarray to test altered gene expression in transgenic plants. Tandem mass spectrometry (LC-MS/MS) of protein extracts from leaf petiole, flower pedicel and their AZ tissues will be used to identify the proteome of the AZ before and during abscission. AZ-specific gene promoters will be used in stably transformed tomato plants to reduce non-target phenotypes. The bean pod mottle virus (BPMV) plasmid vectors will be used for VIGS analysis in soybean. Expected Contribution: Our study will provide new insights into the regulation of ethylene-induced abscission by further revealing the role of key regulators in the process. This will permit development of novel techniques for manipulating leaf and flower abscission, thereby improving the postharvest performance of agriculturally important crops.
\end{abstract}


Summary Sheet

PubType IS only Joint US only

Reviewed 2 42 


\section{CONTRIBUTION OF COLLAORATION:}

All RNA sequencing results for tomato and soybean have been shared between the Israeli and US partners, and the analyses of the results have been performed by a combined effort. Information and ideas about gene regulation in abscission in both tomato and soybean are discussed regularly via email. Partly due to budget restrictions, we were unable to meet in Israel or the US. However, all collaborating investigators met and discussed accomplishments, planned experiments and objectives in August 2014 in Brisbane, Australia at the Abscission Symposium organized as part of the $29^{\text {th }}$ International Horticultural Congress (IHC2014). Moreover, we have published several joint publications. 


\section{ACHIEVEMENTS: Final report (2013-2015)}

This BARD project, US-4571-12C, is a continuation of an earlier project that focused on tomato abscission. The current project continues research on tomato abscission and adds research on soybean, an important crop in the USA.

\section{TOMATO ABSCISSION}

\section{Custom abscission zone-specific microarray}

A primary objective for this granting period was to use transcriptome sequencing to prepare a custom abscission-specific tomato microarray to examine transgenic plants with altered abscission phenotypes. We completed this objective and have published results related to its preparation (Sundaresan et al., 2015, Front. Plant Sci. 6 and PhD thesis, 2016). RNA-seq was performed on a pool of cDNAs sampled during the abscission process from tomato FAZ and LAZ, generating 37.92 and 39.78 million high quality 73-bp paired end reads. AZ-specific transcript information and available tomato gene models were utilized to design a customized AZ-specific microarray chip. A $4 \times 180 \mathrm{~K}-60$ mer oligonucleotide microarray, comprised of 180,880 probes was designed using the transcripts derived from the pooled RNA-seq analysis and known abscission-related transcripts, transcripts from the Solanaceae (Sol) genomics database, NCBI databases and Agilent control and random probe sets. The unique design of this chip allows us to quantify accurately global changes in the transcriptome of tomato AZs during the abscission process. Additionally, in this chip the probes were designed in both sense and antisense orientations, which will enable future analyses of expression profiles of naturally occurring antisense transcripts (NATs) in the AZs.

\section{Functional analysis of selected genes}

The homozygous transgenic plants (T3 generation lines) for tomato abscission polygalacturonase (TAPG) promoter::antisense KD1 and TAPG promoter::antisense tomato proline rich protein (TPRP) constructs, that displayed delayed abscission phenotypes with Viral-Induced Gene Silencing (VIGS) (Jiang's lab), were examined further using microarray and qPCR. Our initial microarray results indicate that a lot of genes are differentially expressed during abscission in the transgenic plants compared to the nontransgenic, wild type plants. In 2015 we published our results on the silencing of KD1 in tomato using the 35S::RNAi KD1 construct (Ma et al, 2015, Plant Physiol., 167:844-853). Suppression of KD1 expression delayed pedicel abscission. A more detailed bioinformatics analysis using our custom tomato abscission microarray (Sundaresan et al., 2015, Front. Plant Sci. 6) is in progress. In addition, we are doing the same with the 35S::RNAi TPRP constructs that also showed a delayed abscission phenotype.

\footnotetext{
Abscission zone (AZ) transcriptome analysis using our customized microarray

We used our newly designed customized microarray (Sundaresan et al., 2015, Front. Plant Sci. 6) to carry out expression profiling of genes linked to abscission. The microarray analysis included flower AZ sampled at $0,2,4,8,14 \mathrm{~h}$ after flower removal and leaf AZ sampled at 0,24, 48, 72, $96 \mathrm{~h}$ after leaf deblading. The results show that a large number of genes were up- or down-regulated in the flower AZ (FAZ) and leaf AZ (LAZ). Several auxin-related genes, Aux/IAA, PIN, and Small Auxin-Up RNA $(S A U R)$, shared a common pattern of down regulation during abscission, with various degrees of expression levels. A few auxin-related genes were expressed specifically in the FAZ or the LAZ. Several ethylene biosynthesis genes, abscission-related genes such as Cellulases and Pathogenesis-Related $(P R)$, and transcription factors genes were up-regulated, while most of the Ethylene Response Factor $(E R F)$ and 1-Amino-Cyclopropane-1-Carboxylic acid (ACC) Synthase (ACS) families were down-regulated during abscission. The analyses revealed that the FAZ and LAZ share similarities but also exhibit differences in gene expression patterns in the execution of abscission. Using the customized AZ-microarray chip, we also identified naturally occurring antisense transcripts. Approximately 5,000 probes detected differential expression in the antisense orientation in both the FAZ and LAZ, indicating their possible significant involvement in abscission. Overall, this study provides new insights into the regulation of early abscission events.
} 
The customized AZ-microarray chip is also being used to continue studies of the previously identified asymmetry between the distal and proximal sides of the AZ and the role of the LX ribonuclease in abscission. Transcriptomic comparison of the proximal and distal sides of the AZ revealed significant differences which indicate that asymmetry in the $\mathrm{AZ}$ exists even before the abscission execution phase is induced. Transcriptomic comparison of AZs from tomato wild type and LX-inhibited transgenic lines revealed significant differences in gene expression, which are currently being investigated.

\section{New transformations of tomato}

We have transformed tomato with RNAi constructs designed to suppress the tomato INFLORESENCE DEFICIENT IN ABSCISSION 1 (IDA1), a nematode IDA (discussed below), and the tomato ETHYLENEINSENSITIVE 2 (EIN2) gene. We currently have multiple events for the SIIDA1 RNAi construct and one line for the nematode IDA RNAi, but have not yet been successful with the SIEIN2 RNAi constructs. Problems with getting EIN2 RNAi lines were not unexpected. We had a similar problem in the Tucker lab 20 years ago when we tried to get transgenic tomato with mutated ethylene receptor. It's our interpretation that if ethylene responses are fully blocked in tomato, regeneration of whole plants from transformed callus is also blocked. Nonetheless, transgenic RNAi plants that suppress the tomato SIIDA1 and the nematode MiIDA1 will be used to examine the role of the tomato IDA in abscission, senescence and the MiIDA1 lines to examine infection of tomato roots by the root-knot nematode (discussed below).

\section{ARABIDOPSIS ABSCISSION}

\section{Continued efforts to elucidate the role of cytosolic pH changes during abscission}

We demonstrated that the cytosolic $\mathrm{pH}$ in the $\mathrm{AZ}$ cells becomes alkaline during abscission, and we hypothesized that various AZ-specific transporter genes may be involved in this $\mathrm{pH}$ regulation (Sundaresan et al., 2015, J Exp. Bot. 66:1355-1368). To evaluate our hypothesis we ordered 35 Arabidopsis SALK mutant lines with T-DNA insertions in genes that were up-regulated in the AZ and were predicted to regulate the cellular $\mathrm{pH}$ (Meir's lab). After initial analysis we chose 4 genes that displayed a strong delayed abscission phenotype (Fig. 1, red bars) that appear to confirm our hypothesis.

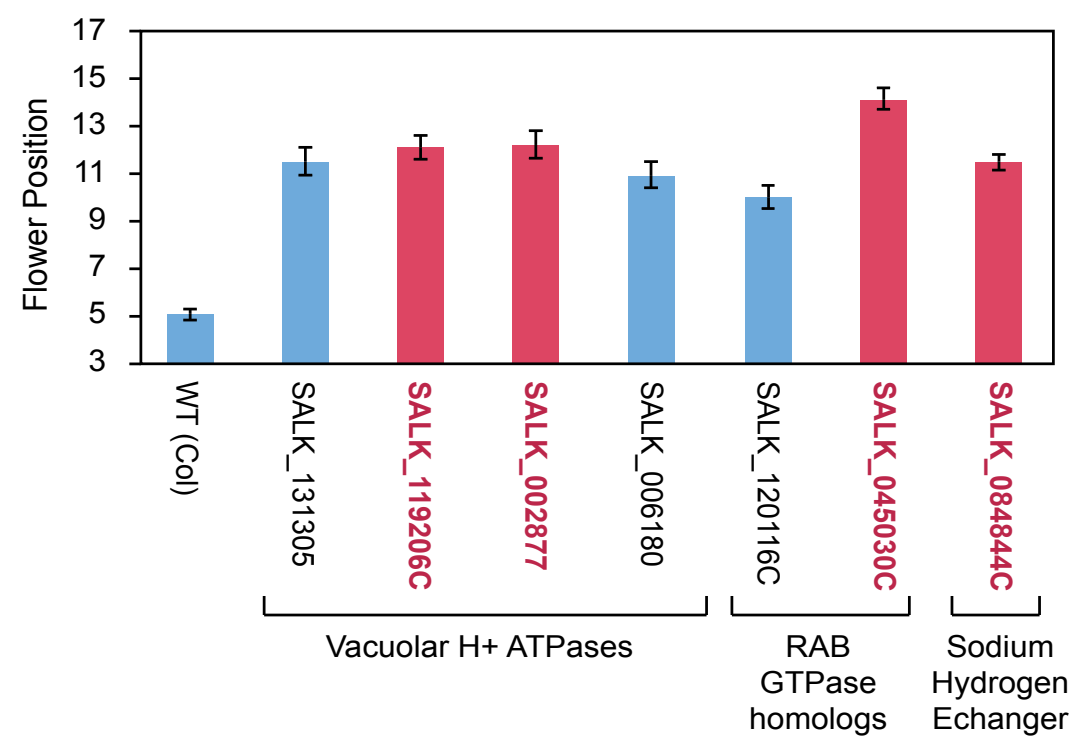

Figure 1. Flower organ abscission in WT Columbia and SALK tDNA mutants

We subsequently selected homozygous lines for these mutant genes and confirmed the delayed abscission phenotypes for these mutants (Fig. 2). The homozygous plants are currently being examined further for cytosolic $\mathrm{pH}$ changes in the $\mathrm{AZ}$ cells using a $\mathrm{pH}$-sensitive and intracellularly-trapped dye, 2',7'-bis-(2- 
carboxyethyl)-5(and-6)-carboxyfluorescein-acetoxymethyl ester derivative (BCECF-AM) and confocal microscopy. In the future we will: (1) examine the effect of the mutations on expression of AZ-specific genes compared to WT, and (2) examine changes in cytosolic $\mathrm{pH}$ in the AZ cells using genetically encoded $\mathrm{pH}$-sensitive sensors.

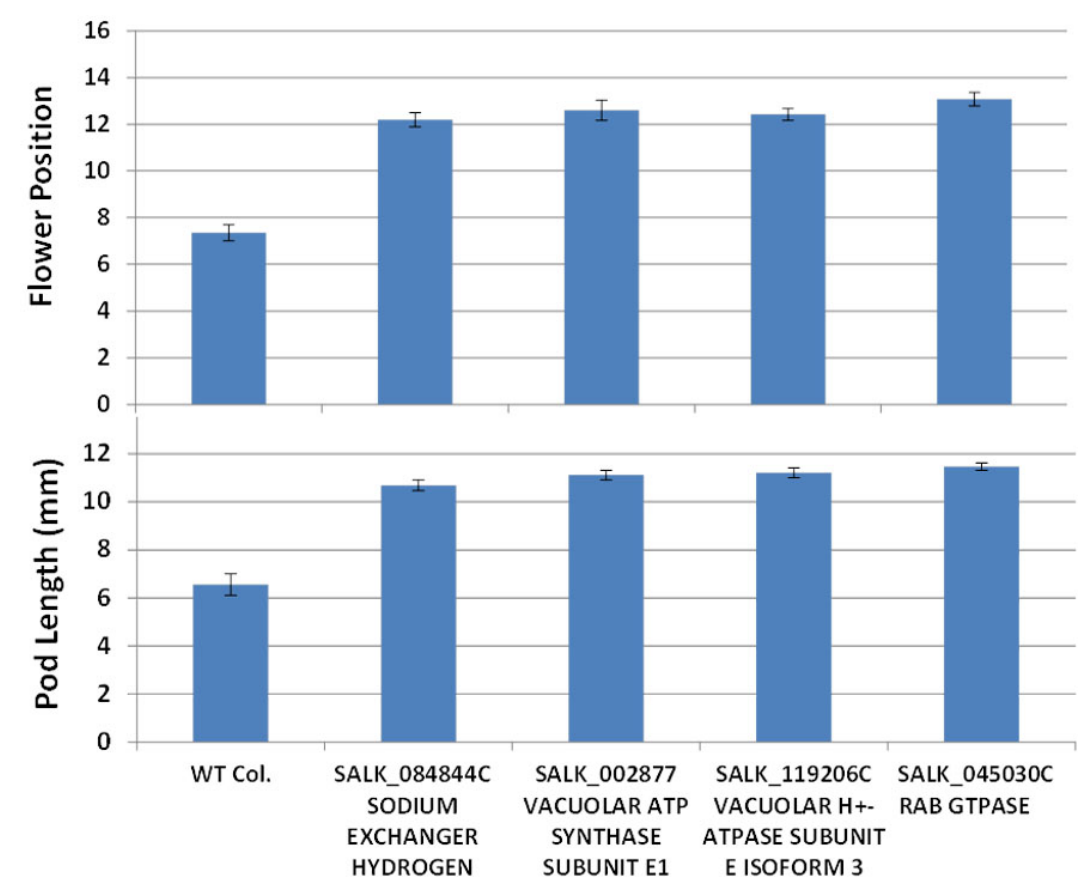

Figure 2. The position of flowers in the inflorescence and the pod length at the time of floral organ abscission.

\section{SOYBEAN ABSCISSION}

Soybean, Glycine max, is an important crop in the USA. The primary objectives for the soybean project were to expand the knowledge base for soybean abscission and transfer knowledge about model systems like tomato and Arabidopsis to soybean. The initial thrusts of our research were: (1) to acquire and analyze the soybean AZ transcriptome using RNA sequencing, and (2) to identify a role in tomato and soybean for the Arabidopsis $I D A$ signaling path, which regulates floral organ abscission in Arabidopsis.

\section{Abscission transcriptome}

In 2013 we isolated RNA from soybean LAZ and petioles (non-AZ, NAZ) after 0, 12, 24, 48 and $72 \mathrm{~h}$ for three replicate experiments and completed RNA-seq on 30 samples (Tucker's lab). We used the soybean RNAseq and custom tomato microarray results to write a paper together that was publish in 2015 (Kim et al., 2015, Front. Plant Sci. 6). Our analysis led to our conclusion that in addition to cell wall loosening enzymes, abscission requires (or is enhanced by) biosynthesis and secretion of waxes and small proteins that form an extensible boundary layer around separating cells. Moreover, we proposed that abscission of plant organs is an adaptation of the primal process of organogenesis occurring in the meristem. In addition, we published results that examined changes in soybean transcription factors expressed during soybean leaf abscission (Kim et al., 2016, Front. Plant Sci. 6). In this study we identified several transcription factors and regulatory processes associated with organ polarity and cell fate, which further supported our proposal that abscission is an adaptation of the primal process of organogenesis occurring in the meristem. 


\section{Role of IDA in soybean and tomato abscission}

Virus-induced gene silencing (VIGS) analysis

$I D A$-like genes were identified in tomato and soybean, and their expression in abscission determined by qPCR. IDA-like genes were up-regulated in both tomato and soybean. The tomato VIGS system developed by Cai-Zhong Jiang at UC Davis was used to suppress expression of IDA genes in tomato organ abscission. No delay of flower or leaf abscission was observed in plants expressing the IDA-VIGS construct. A bean pod mottle virus, which was developed for VIGS in soybean, was acquired from Steve Whitham (Iowa State University) and the VIGS protocol was established in Tucker's lab. In 2013 constructs were prepared to suppress expression of the soybean IDAs to determine if IDA signaling plays a role in soybean leaf abscission. No abscission phenotype was observed, but we did observe a slight delay in leaf senescence. qPCR was used to examine the expression of IDA-like and HAESA-like receptor genes during soybean senescence. Surprisingly, expression of these genes was very strong during leaf senescence. We believe IDA signaling may play an important role in senescence and have begun experiments to examine this role further. Therefore, because $I D A$-like gene expression was abscissionspecific and we saw an IDA link to senescence, we decided it was prudent to prepare stably transformed tomato with an SIIDA1 RNAi construct. Suppression of SIIDA1 throughout the tomato plant will provide a better understanding of the role of IDAs in abscission, senescence and overall plant development. We currently have several first generation tomato lines (events) for the SIIDA1 RNAi transformants and will soon begin our analysis of the plants.

\section{Root-knot nematode (M. incognita) IDA}

As a corollary to our abscission research on IDA, we are examining the role of a recently identified IDAlike gene in a root-knot nematode (Meloidogyne incognita), which infects tomato, soybean, and several other important agricultural crops. We have demonstrated that the nematode IDA, as well as the soybean IDA, can restore a normal abscission phenotype to the Arabidopsis ida mutant. We concluded that both the nematode and the soybean IDAs can function as an IDA signal in Arabidopsis. We have generated transgenic Arabidopsis that overexpress the nematode gene and suppress its expression (RNAi construct). Overexpression of the nematode $I D A$ enhances floral organ abscission and alters root growth. Moreover, MiIDA1 RNAi expression in Arabidopsis roots inhibited the number of nematode induced galls and reduced the size of the galls that formed on the roots. This suggests that the nematode IDA-like gene plays an important role in establishing its feeding structure on roots. In addition to Arabidopsis, we have begun similar RNAi experiments in soybean and tomato using a hairy root transformation protocol. 


\section{CHANGES TO ORIGINAL RESEARCH PLAN:}

1) Because the funding was less than requested, salaries and supplies for graduate students and postdocs are being supplemented with in-house funding. The proposal objectives were slightly altered to address mission requirement for in-house funding. In particular, the mission for Dr. Tucker's inhouse funding supports research on nematodes. Interestingly, genes expressed in soybean abscission are also up-regulated during nematode infection of soybean roots, and may share a common form of regulation. Thus, while we examine a role for gene regulation in abscission, we are complimenting the BARD research with research to determine regulation of host gene expression during nematode infection.

2) Since we found a strong expression of the IDA gene in soybean leaf senescence, we will continue to pursue this line of research.

3) We originally proposed to perform proteomics (tandem mass spectrometry) on tomato AZ protein. Funding was insufficient to perform this objective.

4) Regarding the interesting results showing the involvement of $\mathrm{pH}$ changes in the AZs of tomato, wild rocket and Arabidopsis, we have expanded the research on the role of $\mathrm{pH}$ changes in the abscission process more than originally proposed. Thus, we examined the SALK Arabidopsis mutants for delayed abscission phenotypes (Fig. 1 and Fig. 2), and we plan to further expand this line of research in the future. 


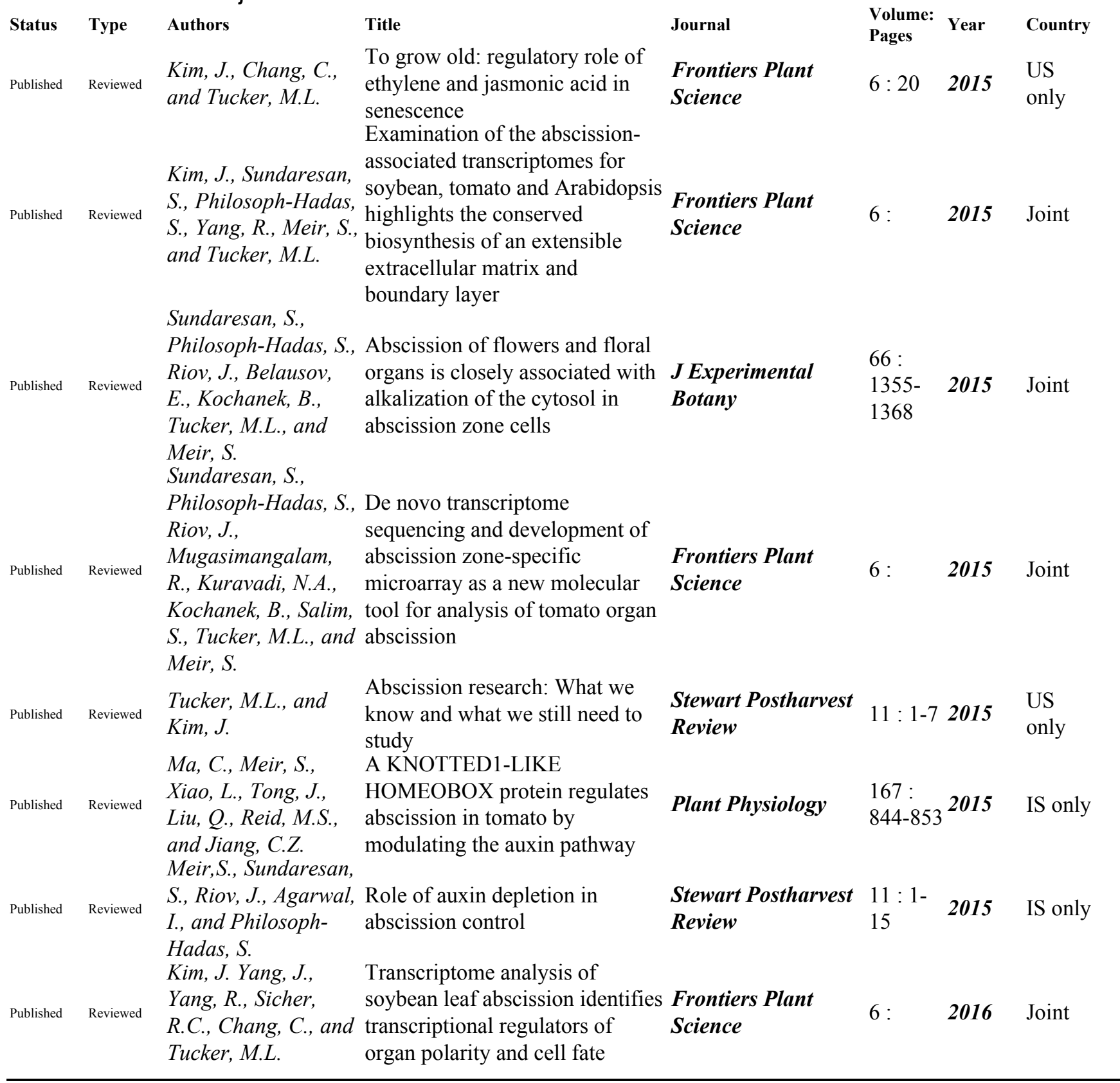




\section{APPENDIX: Abstracts and Conference Presentations:}

1. Sundaresan, S., Philosoph-Hadas, S., Kochanek, B., Belausov, E., Riov, J. and Meir, S. (2013). Ethylene- enhanced flower abscission is associated with specific increased $\mathrm{pH}$ of the cytosol in abscission zone cells of Arabidopsis, wild rocket and tomato flowers. Abstract and lecture in the 21st Conference of the International Plant Growth Substances association (IPGSA), Shanghai, China, Abstract No. C-16, p. 177 (Lecture presentation).

2. Sundaresan, S., Philosoph-Hadas, S., Riov, J., Kochanek, B., Mugasimangalam, R. and Meir, S. (2014). Abscission regulatory networks: a transcriptomic analysis using NGS and a novel tomato flower and leaf abscission zone customized microarray. Abstract and poster in the 7th Congress of the federation of the Israel societies of experimental biology (FISEB, ILANIT), Eilat, Israel, Abstract No. PB-71, p. 36 (Poster presentation).

3. Sundaresan, S., Philosoph-Hadas, S., Riov, J., Kochanek, B., Mugasimangalam, R. and Meir, S. (2014). Transcriptome Analysis of the Tomato Flower and Leaf Abscission Zones, using a customized abscission zone microarray. Abstract and lecture in the 29th International Horticultural Congress (IHC2014), Brisbane, Australia, Abstract No. 00751 (Lecture presentation).

4. Meir, S., Sundaresan, S., Ma, C., Philosoph-Hadas, S., Riov, J., Lers, A., Kochanek, B., Tucker, M., Reid, M.S., Jiang, C-Z., (2014). New Insights and Approaches for Elucidating Regulation of Organ Abscission. Abstract and lecture in the 29th International Horticultural Congress (IHC2014), Brisbane, Australia, Abstract No 00625 (Lecture presentation).

5. Lers, A., Bar-Dror, T., Dermastia, M., Kladnik, A., Sundaresan, S., Meir, S., Burd, S., PhilosophHadas, Magada Tusek, Z., Marusa Pompe, N., (2014). Asymmetry Between the Distal and Proximal Sides of the Abscission Zone: Characterization, Functional Significance and the Involvement of Programmed Cell Death. Abstract and lecture in the 29th International Horticultural Congress (IHC2014), Brisbane, Australia, Abstract No. 04818 (Lecture presentation).

6. Kim, J., Binder, B.M., Patterson, S., Chang, C. and Tucker, M.L. (2014). Independent and interdependent regulation of floral organ abscission by ET and JA. Abstract and lecture 29th International Horticultural Congress (IHC2014) in Brisbane Australia, Abstract No. 01869 (Lecture presentation).

7. Tucker, M.L., Kim, J., Sundaresan, S., Yang, R., Chang, C., Meir, S., Philosoph-Hadas, S., Lers, A., Reid, M. and Jiang, C.-Z. (2014). A functional study of IDA-like genes in soybean and tomato. Abstract and lecture 29th International Horticultural Congress (IHC2014) in Brisbane Australia, Abstract No. 02748 (Lecture presentation).

8. Kim, J., Yang, R., Chang, C. and Tucker, M.L. (2015). A functional study of a root knot nematode IDA-like (IDL) peptide putatively secreted into the host plant. Mid-Atlantic Plant Molecular Biology Society (Lecture presentation).

9. Kim, J., Yang, R., Chang, C. and Tucker, M.L. (2015). A functional study of a root knot nematode IDA-like (IDL) peptide putatively secreted into the host plant. American Society of Plant Biology (Poster presentation).

10. Sundaresan, S., Philosoph-Hadas, S., Riov, J., Belausov, E., Kochanek, B., Segev, A., and Meir, S. (2015). Abscission of flowers and floral organ is closely associated with alkalization of the cytosol in the abscission zone cells. Abstract and Poster in the Esra Gala memorial Symposium - Israeli society of plant sciences (ISPS), Rehovot, Israel. P.75 (Poster presentation).

11. Sundaresan, S., Philosoph-Hadas, S., Riov, J., Kochanek, B., Salim, S., Agarwal, I., and Meir, S. (2015).Transcriptome profiling of ethylene-related molecular changes occurring in the tomato flower and leaf abscission zones in response to auxin depletion. Abstract and lecture in the 10th International conference on the plant hormone ethylene, Chongqing, China, Session 10-3, P.52 (Lecture presentation). 


\section{Mansucripts in prepration:}

1. Sundaresan, S., Philosoph-Hadas, S., Ma, C., Jiang, C.Z., Riov, J., Mugasimangalam, R., Kochanek, B., Salim, S., Reid, M.S., and Meir, S. (2016). Regulation of the transcriptional network during flower abscission by the tomato hybrid proline-rich protein (THyPRP).

2. Sundaresan, S., Philosoph-Hadas, S., Ma, C., Jiang, C.Z., Riov, J., Mugasimangalam, R., Kochanek, B., Salim, S., Reid, M.S., and Meir, S. (2016). Transcriptional cascade of KNOTTED1-LIKE HOMEOBOX Protein (KD1) in regultion of tomato flower abscission.

3. Sundaresan, S., Agarwal, I., Philosoph-Hadas, S., Riov, J., Mugasimangalam, R. and Meir, S. (2016). Characterization and expression of naturally occurring antisense transcripts in tomato flower and leaf abscission zones.

4, Kim, J., Yang, R., Chang, C., and Tucker, M.L. (in preparation) The root-knot nematode (Meloidogyne incognita) IDA-like peptide rescues the $i d a$ abscission and root phenotypes and its suppression reduces nematode infection of transgenic Arabidopsis roots.

\section{PhD Thesis:}

1. Srivignesh Sundaresan, Regulation of Flower Abscission in Tomato (Solanum lycopersicum) Plants: Functional and Transcriptome Analyses. Submitted February 2016 to Department of Postharvest Science of Fresh Produce, Agricultural Research Organization, The Volcani Center, Bet-Dagan, Israel. 